\title{
Quadrature Formulae for Cauchy Principal Value Integrals of Oscillatory Kind
}

\author{
By G. E. Okecha
}

\begin{abstract}
The problem considered is that of evaluating numerically an integral of the form $f_{-1}^{1} e^{i \omega x} f(x) d x$, where $f$ has one simple pole in the interval $[-1,1]$. Modified forms of the Lagrangian interpolation formula, taking account of the simple pole are obtained, and form the bases for the numerical quadrature rules obtained. Further modification to deal with the case when an abscissa in the interpolation formula is coincident with the pole is also considered. An error bound is provided and some numerical examples are given to illustrate the formulae developed.
\end{abstract}

1. Introduction. The numerical evaluation of finite Fourier integrals of the form

$$
\int_{-1}^{1} e^{i \omega x} f(x) d x, \quad \omega \geqslant 0, i^{2}=-1,
$$

has wide applications in applied mathematics, physics and engineering. If $\omega$ is large, the integrand is highly oscillatory, and classical methods of integration are unsuitable. Here, $f$ is sufficiently smooth, usually analytic, in the range of integration $[-1,1]$.

The earliest numerical method for the treatment of (1.1) is probably due to Filon [6] who approximates $f$ by second-degree polynomials over an even number of subintervals and analytically integrates out the crippling oscillatory factor. Full details of this method are also given in Davis and Rabinowitz [4, Eq. (2.10.2)].

A notable later work is that due to Luke [14], who approximates $f$ by polynomials of degree $\leqslant 10$. Since then, a considerable literature has evolved on the subject, and in this connection one may see [2], [12], [17], [18], [22].

In this paper we are concerned with the evaluation of the integral

$$
I(\tau, \omega)=\int_{-1}^{1} e^{i \omega x} \frac{g(x)}{x-\tau} d x, \quad-1<\tau<1,
$$

where $g$ is analytic for $-1 \leqslant x \leqslant 1$ and $g(\tau) \neq 0$. The integral (1.2) has two practical difficulties - it is oscillatory and has a singularity of Cauchy type; for the latter see [3], [9], [11], [13], [16]. To deal with these pertinent problems, we present a method based on a modified Lagrangian interpolation formula and on properties of orthogonal polynomials.

Received May 21, 1985; revised August 26, 1985, May 27, 1986, and November 7, 1986. 1980 Mathematics Subject Classification (1985 Revision). Primary 65D05, 65D30; Secondary 41A10.

Key words and phrases. Lagrangian interpolation, orthogonal polynomials, oscillatory integrals, Cauchy principal value, Legendre polynomials, trror bound. 
Given the distinct points $x_{1}, x_{2}, \ldots, x_{n}$ such that the values of some function $\rho$ are defined and known at these points, it is known [20] that there exists a unique polynomial $h_{n-1}$ of degree $n-1$ such that $h_{n-1}\left(x_{\kappa}\right)=\rho\left(x_{\kappa}\right), \kappa=1, \ldots, n$. This interpolating polynomial $h_{n-1}$, written in Lagrangian form, is

$$
h_{n-1}(x)=\sum_{\kappa=1}^{n} \rho\left(x_{\kappa}\right) l_{\kappa}(x) \text {, }
$$

where the polynomials $l_{\kappa}$, the Lagrange coefficients, are given by

and

$$
l_{\kappa}(x)=\frac{w_{n}(x)}{w_{n}^{\prime}\left(x_{\kappa}\right)\left(x-x_{\kappa}\right)},
$$

Clearly,

$$
w_{n}(x)=\prod_{\kappa=1}^{n}\left(x-x_{\kappa}\right)
$$

Throughout the rest of this paper we shall assume that the set $\mathbf{X}=\left\{x_{1}, x_{2}, \ldots, x_{n}\right\}$ is the set of zeros of a polynomial of degree $n$ from an orthogonal sequence with respect to a nonnegative weight function $w$ in $(-1,1)$. Therefore, the set $\mathbf{X}$ is distinct.

The basis of our quadrature formulae is a modification of (1.3).

2. Description of Method. Suppose $U_{n}(g ; \cdot)$ is the Lagrange interpolation polynomial of degree $n$ interpolating to $g$ at the element-points of $\mathbf{X}$ and at $\tau$. Then we have

$$
U_{n}(g ; x)=(x-\tau) \sum_{\kappa=1}^{n} \frac{l_{\kappa}(x) g\left(x_{\kappa}\right)}{x_{\kappa}-\tau}+\frac{w_{n}(x) g(\tau)}{w_{n}(\tau)}, \quad x_{\kappa} \neq \tau .
$$

Thus,

$$
g(x)=U_{n}(g ; x)+e_{n}(g ; x)
$$

where $e_{n}(g ; x)$ is the error due to the interpolation formula and is given as

$$
e_{n}(g ; x)=(x-\tau) w_{n}(x) g\left[x, x_{1}, x_{2}, \ldots, x_{n}, \tau\right] .
$$

Here, $g\left[x, x_{1}, x_{2}, \ldots, x_{n}, \tau\right]$ is the $(n+1)$ st divided difference of $g$ corresponding to the abscissae $x, x_{1}, x_{2}, \ldots, x_{n}, \tau$.

From [1, Eq. (25.1.8)],

$$
g\left[x, x_{1}, x_{2}, \ldots, x_{n}, \tau\right]=\frac{1}{2 \pi i} \int_{C} \frac{g(z) d z}{(z-\tau)(z-x) w_{n}(z)},
$$

where $C$ is a contour containing the points $x, x_{1}, x_{2}, \ldots, x_{n}, \tau$ in its interior and $g$ is analytic in the interior of $C$.

Now, multiplying (2.2) through by $e^{i \omega x} /(x-\tau)$ and integrating over $(-1,1)$ in the CPV sense, one gets

$$
I(\tau, \omega)=\sum_{\kappa=1}^{n} \frac{g\left(x_{\kappa}\right)}{\left(x_{\kappa}-\tau\right) w_{n}^{\prime}\left(x_{\kappa}\right)} \int_{-1}^{1} \frac{e^{i \omega x} w_{n}(x) d x}{x-x_{\kappa}}+\frac{g(\tau)}{w_{n}(\tau)} f_{-1}^{1} \frac{e^{i \omega x} w_{n}(x) d x}{x-\tau}
$$

$$
+\int_{-1}^{1} e^{i \omega x} w_{n}(x) g\left[x, x_{1}, x_{2}, \ldots, x_{n}, \tau\right] d x \text {. }
$$


Suppose $M_{n}$, a multiple of $w_{n}$, is the orthogonal polynomial whose zeros are the elements of the set $\mathbf{X}$; then,

$$
I(\tau, \omega)=\sum_{\kappa=1}^{n} \frac{g\left(x_{\kappa}\right) Z_{n}\left(x_{\kappa}, \omega\right)}{\left(x_{\kappa}-\tau\right) M_{n}^{\prime}\left(x_{\kappa}\right)}+\frac{g(\tau) Z_{n}(\tau, \omega)}{M_{n}(\tau)}+E_{n}(\tau, \omega),
$$

where

$$
\begin{gathered}
Z_{n}(\alpha, \omega)=\int_{-1}^{1} \frac{e^{i \omega x} M_{n}(x) d x}{x-\alpha}, \quad-1<\alpha<1, \\
E_{n}(\tau, \omega)=\frac{1}{2 \pi i} \int_{C} \frac{g(z) d z}{(z-\tau) M_{n}(z)} \int_{-1}^{1} \frac{e^{i \omega x} M_{n}(x) d x}{z-x},
\end{gathered}
$$

and $E_{n}(\tau, \omega)$ is the error due to the quadrature rule. In Section 7 we shall obtain a bound for $E_{n}(\tau, \omega)$ for a particular $M_{n}$. By construction, $E_{n}(\tau, \omega)=0$ whenever $g$ is a polynomial of degree $\leqslant n$.

3. Evaluating $Z_{n}(\alpha, \omega)$. We describe two ways of evaluating $Z_{n}(\alpha, \omega)$.

(i) If $\alpha$ is not a zero of $M_{n}$ and $Z_{n}(\alpha, \omega)$ is not known analytically, perhaps the most convenient way to evaluate $Z_{n}(\alpha, \omega)$ is from a recursion equation which can be established as follows.

It is common knowledge that the orthogonal polynomials $M_{r}(x)$ satisfy a relation of the form

$$
M_{r+1}(x)=\left(A_{r}+B_{r} x\right) M_{r}(x)-C_{r} M_{r-1}(x), \quad r=0,1, \ldots,
$$

with $B_{r}>0, C_{r}>0, M_{0}=1, M_{1}=A_{0}+B_{0} x, M_{-1}=0$.

In view of (2.7) and (3.1) it can be shown that

$$
\begin{aligned}
Z_{r+1}(\alpha, \omega)=\left(A_{r}+B_{r} \alpha\right) Z_{r}(\alpha, \omega)-C_{r} Z_{r-1}(\alpha, \omega)+B_{r} \tilde{Z}_{r}(\omega) & \\
r & =0,1, \ldots,
\end{aligned}
$$

where

$$
\tilde{Z}_{r}(\omega)=\int_{-1}^{1} e^{i \omega x} M_{r}(x) d x
$$

and $\tilde{Z}_{r}(\omega)$ may be evaluated analytically or can be sufficiently approximated using any rule designed for oscillatory functions, e.g. [2], [18].

The inhomogeneous three-term recurrence relation (3.2) is stable in the forward direction provided that $|\alpha|<1$; otherwise, instability sets in and a procedure such as that given by Miller [15, p. xvii] must be used. The starting values are

$$
Z_{0}(\alpha, \omega)=\int_{-1}^{1} \frac{e^{i \omega x}}{x-\alpha} d x
$$

and, as is easily seen from (3.2),

$$
Z_{1}(\alpha, \omega)=\left(A_{0}+\alpha\right) Z_{0}(\alpha, \omega)+2 B_{0} \frac{\sin \omega}{\omega},
$$

where $A_{0}, B_{0}$ are the coefficients in $M_{1}(x)=A_{0}+B_{0} x$. 
Let $z$ be complex, $z=x+i y, i=\sqrt{-1}$. For $z \notin(-1,1)$, set

$$
q(z)=\int_{-1}^{1} \frac{\beta(x)}{z-x} d x
$$

where $\beta$ is a smooth function of $x$ in $[-1,1]$. Further, if $z \in(-1,1)$, with the convention that $q(z)=\frac{1}{2}[q(z+0 i)-q(z-0 i)],-1<z<1$, it is easy to see that

$$
q(z)=\int_{-1}^{1} \frac{\beta(x)}{z-x} d x .
$$

Using this in [8, Eq. 2.641, nos. 1 and 2], we obtain

$$
\begin{aligned}
\operatorname{Re}\left[Z_{0}(\alpha, \omega)\right]= & \int_{-1}^{1} \frac{\cos \omega x d x}{x-\alpha}=\cos \omega \alpha \operatorname{Ci}\left(u_{1}\right)-\sin \omega \alpha \operatorname{Si}\left(u_{1}\right) \\
& +\sin \alpha \omega \operatorname{Si}\left(u_{2}\right)-\cos \alpha \omega \operatorname{Ci}\left(u_{2}\right), \\
\operatorname{Im}\left[Z_{0}(\alpha, \omega)\right]= & \int_{-1}^{1} \frac{\sin \omega x d x}{x-\alpha}=\cos \alpha \omega \operatorname{Si}\left(u_{1}\right)+\sin \alpha \omega \operatorname{Ci}\left(u_{1}\right) \\
& -\cos \alpha \omega \operatorname{Si}\left(u_{2}\right)-\sin \alpha \omega \operatorname{Ci}\left(u_{2}\right),
\end{aligned}
$$

where

$$
u_{1}=\omega(1-\alpha), \quad u_{2}=-\omega(1+\alpha),
$$

and $\mathrm{Ci}$ and $\mathrm{Si}$ are the cosine and sine integrals, respectively.

(ii) If $\alpha$ is a zero of $M_{n}(x)$, then $Z_{n}(\alpha, \omega)$ may be expressed as follows on using the Christoffel-Darboux identity,

$$
\begin{gathered}
Z_{n}(\alpha, \omega)=\sum_{m=0}^{n-1} d_{m} M_{m}(\alpha) \tilde{Z}_{m}(\omega), \\
d_{m}=\frac{-k_{n+1}}{k_{n}} \cdot \frac{h_{n}}{h_{m}} \cdot \frac{1}{M_{n+1}(\alpha)},
\end{gathered}
$$

where

$$
h_{n}=\int_{-1}^{1} w(x) M_{n}^{2}(x) d x
$$

and $k_{n}$ is the coefficient of $x^{n}$ in $M_{n}(x)$. The pairs $\left(h_{r}, k_{r}\right)$ have been tabulated [1] for some orthogonal polynomials. Note, however, that the method of (3.2) is still valid in this case.

4. Application with the Legendre Polynomials. We assume now that the orthogonal polynomials $M_{r}(x)$ are the Legendre polynomials $P_{r}(x)$ whose zeros now form the set $\mathbf{X}=\left\{x_{1}, x_{2}, \ldots, x_{r}\right\}$. Therefore,

$$
\begin{gathered}
Z_{n}(\alpha, \omega)=\int_{-1}^{1} \frac{e^{i \omega x} P_{n}(x) d x}{x-\alpha}, \quad-1<\alpha<1, \\
M_{n}^{\prime}(x)=P_{n}^{\prime}(x)=\frac{n x P_{n}(x)-n P_{n-1}(x)}{x^{2}-1}, \quad x \neq \pm 1 .
\end{gathered}
$$

Thus from (2.6) we have the approximate rule

$$
I_{n}(\tau, \omega)=\sum_{\kappa=1}^{n} \frac{\left(1-x_{\kappa}^{2}\right) g\left(x_{\kappa}\right) Z_{n}\left(x_{\kappa}, \omega\right)}{n P_{n-1}\left(x_{\kappa}\right)\left(x_{\kappa}-\tau\right)}+\frac{g(\tau) Z_{n}(\tau, \omega)}{P_{n}(\tau)}, \quad x_{\kappa} \neq \tau .
$$


The Legendre polynomials $P_{r}(x)$ satisfy the recurrence relation

$$
(r+1) P_{r+1}(x)-(2 r+1) x P_{r}(x)+r P_{r-1}(x)=0,
$$

and from (4.1) and (4.4) we obtain [cf. (3.2)]

$$
\begin{array}{r}
(r+1) Z_{r+1}(\alpha, \omega)=(2 r+1) \alpha Z_{r}(\alpha, \omega)-r Z_{r-1}(\alpha, \omega)+(2 r+1) \tilde{Z}_{r}(\omega), \\
r=0,1, \ldots,
\end{array}
$$

where

$$
\begin{gathered}
\operatorname{Re}\left[\tilde{Z}_{r}(\omega)\right]=\int_{-1}^{1} \cos \omega x P_{r}(x) d x=2(-1)^{k} j_{2 k}(\omega), \\
r=2 k, k=0,1, \ldots, \\
\operatorname{Im}\left[\tilde{Z}_{r}(\omega)\right]=\int_{-1}^{1} \sin \omega x P_{r}(x) d x=2(-1)^{k} j_{2 k+1}(\omega), \\
r=2 k+1, k=0,1, \ldots,
\end{gathered}
$$

and where $j_{\kappa}(x)$ are the spherical Bessel functions of the first kind, which can be evaluated as in [1, Eq. (10.5)].

In view of (3.5),

$$
\begin{aligned}
& \operatorname{Re}\left[Z_{1}(\alpha, \omega)\right]=\frac{2 \sin \omega}{\omega}+\alpha \operatorname{Re}\left[Z_{0}(\alpha, \omega)\right], \\
& \operatorname{Im}\left[Z_{1}(\alpha, \omega)\right]=\alpha \operatorname{Im}\left[Z_{0}(\alpha, \omega)\right],
\end{aligned}
$$

with $Z_{0}(\alpha, \omega)$ as in (3.8), (3.9).

Since $x_{\kappa}$ is a zero of $P_{n}(x)$, then from (3.11), (3.12) we may write

$$
Z_{n}\left(x_{\kappa}, \omega\right)=-\frac{1}{(n+1) P_{n+1}\left(x_{\kappa}\right)} \sum_{m=0}^{n-1}(2 m+1) P_{m}\left(x_{\kappa}\right) \tilde{Z}_{m}(\omega) \text {. }
$$

5. Extension. We now assume that an element of $\mathbf{X}$, say $x_{s}$, coincides with $\tau$, i.e.,

$$
x_{s}=\tau, \quad \mathbf{X}=\left\{x_{1}, x_{2}, \ldots, \tau, \ldots, x_{n}\right\} .
$$

Then, with $\tilde{U}_{n}(g ; \cdot)$ the Lagrangian polynomial which interpolates to $g$ at $x_{1}, x_{2}, \ldots, x_{s}, \ldots, x_{n}$ and to $g^{\prime}$ at $x_{s}$, we have as in [3],

$$
\begin{aligned}
\tilde{U}_{n}(g ; x)= & (x-\tau) \sum_{\substack{\kappa=1 \\
\kappa \neq s}}^{n} \frac{l_{\kappa}(x) g\left(x_{\kappa}\right)}{x_{\kappa}-\tau}+\left[1-l_{s}^{\prime}(\tau)(x-\tau)\right] l_{s}(x) g(\tau) \\
& +(x-\tau) l_{s}(x) g^{\prime}(\tau) .
\end{aligned}
$$

Thus,

$$
g(x)=\tilde{U}_{n}(g ; x)+e_{n}(g ; x) .
$$

Again, multiplying (5.2) through by $e^{i \omega x} /\left(x-x_{s}\right)$, integrating over $(-1,1)$, and assuming as previously that the polynomials $M_{r}$ are the Legendre polynomials $P_{r}$, we have the approximate rule

$$
\tilde{I}_{n}(\tau, \omega)=\sum_{\substack{\kappa=1 \\ \kappa \neq s}}^{n} \frac{\left(1-x_{\kappa}^{2}\right) g\left(x_{\kappa}\right) Z_{n}\left(x_{\kappa}, \omega\right)}{n P_{n-1}\left(x_{\kappa}\right)\left(x_{\kappa}-\tau\right)}+g(\tau) Q_{s}+H_{s} g^{\prime}(\tau), \quad \tau=x_{s}
$$


where

$$
\begin{gathered}
H_{s}=\frac{\left(1-x_{s}^{2}\right) Z_{n}\left(x_{s}, \omega\right)}{n P_{n-1}\left(x_{s}\right)}, \\
Q_{s}=\int_{-1}^{1} \frac{l_{s}(x)}{x-x_{s}}\left[1-l_{s}^{\prime}\left(x_{s}\right)\left(x-x_{s}\right)\right] e^{i \omega x} d x \\
=\frac{\left(1-x_{s}^{2}\right)}{n P_{n-1}\left(x_{s}\right)} \int_{-1}^{1} \frac{P_{n}(x) e^{i \omega x} d x}{\left(x-x_{s}\right)^{2}}-l_{s}^{\prime}\left(x_{s}\right) H_{s} .
\end{gathered}
$$

From the relations

$$
\begin{gathered}
\left(1-x^{2}\right) P_{n}^{\prime \prime}(x)-2 x P_{n}^{\prime}(x)+n(n+1) P_{n}(x)=0, \\
\left(x^{2}-1\right) P_{n}^{\prime}(x)=n x P_{n}(x)-n P_{n-1}(x),
\end{gathered}
$$

and with the help of the L'Hospital rule we find

$$
l_{s}^{\prime}\left(x_{s}\right)=\frac{x_{s}}{1-x_{s}^{2}} .
$$

Using the Christoffel-Darboux identity,

$$
\frac{P_{n}(x)}{\left(x-x_{s}\right)^{2}}=-\frac{1}{(n+1) P_{n+1}\left(x_{s}\right)} \sum_{m=0}^{n-1} \frac{(2 m+1) P_{m}\left(x_{s}\right) P_{m}(x)}{x-x_{s}} .
$$

In view of this,

$$
\int_{-1}^{1} \frac{P_{n}(x) e^{i \omega x} d x}{\left(x-x_{s}\right)^{2}}=-\frac{1}{(n+1) P_{n+1}\left(x_{s}\right)} \sum_{m=0}^{n-1}(2 m+1) P_{m}\left(x_{s}\right) Z_{m}\left(x_{s}, \omega\right)
$$

Thus,

$$
\begin{aligned}
Q_{s}= & \frac{\left(x_{s}^{2}-1\right)}{n(n+1) P_{n-1}\left(x_{s}\right) P_{n+1}\left(x_{s}\right)} \\
& \cdot \sum_{m=0}^{n-1}(2 m+1) P_{m}\left(x_{s}\right) Z_{m}\left(x_{s}, \omega\right)+\left(\frac{x_{s}}{x_{s}^{2}-1}\right) H_{s} .
\end{aligned}
$$

Introducing (5.4) and (5.9) into (5.3), we obtain the approximate rule. Again, by construction, this rule is exact whenever $g$ is a polynomial of degree $\leqslant n$.

Consider, in particular, the case $\tau=0$. Let $n$ be odd; then $x_{(n+1) / 2}=0$. With $x_{s}=x_{(n+1) / 2}=0$, it follows from (5.3) and (5.9) that

$$
\tilde{I}_{n}(0, \omega)=\sum_{\substack{\kappa=1 \\ \kappa \neq s}}^{n} \frac{\left(1-x_{\kappa}^{2}\right) g\left(x_{\kappa}\right) Z_{n}\left(x_{\kappa}, \omega\right)}{n P_{n-1}\left(x_{\kappa}\right) x_{\kappa}}+g(0) Q_{s}+H_{s} g^{\prime}(0),
$$

where

$$
Q_{s}=\frac{-1}{n(n+1) P_{n-1}(0) P_{n+1}(0)} \sum_{m=0}^{n-1}(2 m+1) P_{m}(0) Z_{m}(0, \omega)
$$

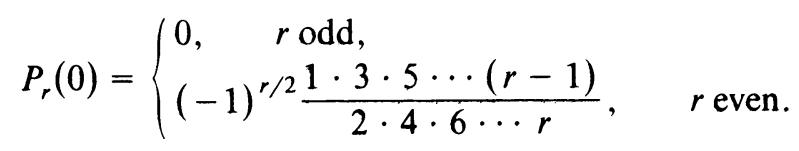


We now describe a method closely related to that in Section 2.

For a given $n$, let $g$ be approximated by the Lagrange interpolation polynomial $U_{n-1}^{*}(g ; \cdot)$ of degree $n-1$. Then we may write

$$
U_{n-1}^{*}(g ; x)=\sum_{\kappa=1}^{n} l_{\kappa}(x) g\left(x_{\kappa}\right)
$$

where $x_{\kappa} \in \mathbf{X}$ as previously defined.

Thus,

$$
g(x)=U_{n-1}^{*}(g ; x)+e_{n-1}^{*}(g ; x)
$$

with the error $e_{n-1}^{*}(g ; x)=w_{n}(x) g\left[x, x_{1}, x_{2}, \ldots, x_{n}\right]$. Again we multiply (5.14) through by $e^{i \omega x} /(x-\tau)$ and integrate over $(-1,1)$ in the CPV sense to obtain the quadrature formula

$$
I(\tau, \omega)=\sum_{\kappa=1}^{n} g\left(x_{\kappa}\right) \frac{\left[Z_{n}\left(x_{\kappa}, \omega\right)-Z_{n}(\tau, \omega)\right]}{\left(x_{\kappa}-\tau\right) M_{n}^{\prime}\left(x_{\kappa}\right)}+E_{n}^{*}(\tau, \omega), \quad x_{\kappa} \neq \tau
$$

where

$$
E_{n}^{*}(\tau, \omega)=\frac{1}{2 \pi i} \int_{C} \frac{g(z) d z}{M_{n}(z)} \cdot \int_{-1}^{1} \frac{e^{i \omega x} M_{n}(x) d x}{(z-x)(x-\tau)}
$$

Furthermore, if a zero of $M_{n}$, say $x_{s}$, coincides with $\tau$, i.e., $x_{s}=\tau$, then it can be readily shown from $(5.15)$ that

$$
I(\tau, \omega)=\sum_{\substack{\kappa=1 \\ \kappa \neq s}}^{n} g\left(x_{\kappa}\right) \frac{\left[Z_{n}\left(x_{\kappa}, \omega\right)-Z_{n}(\tau, \omega)\right]}{\left(x_{\kappa}-\tau\right) M_{n}^{\prime}\left(x_{\kappa}\right)}+\frac{g(\tau) Z_{n}^{\prime}(\tau, \omega)}{M_{n}^{\prime}(\tau)}+E_{n}^{*}(\tau, \omega) .
$$

The formulae (5.15) and (5.17) are exact whenever $g$ is a polynomial of degree $\leqslant n-1$.

6. A Stable Algorithm. The quadrature rules given by (2.6), (4.3), (5.3), (5.15), and (5.17) are numerically unstable when $\tau$ is close to one of the points $x_{i}$; to avoid this, the following algorithm has been proposed [7], [16].

Let $P_{n-1}(g ; \cdot)$ be the polynomial of degree $\leqslant n-1$ interpolating $g$ at the zeros $x_{i}$ of $M_{n}$, written in the form

$$
P_{n-1}(g ; x)=\sum_{\kappa=0}^{n-1} a_{\kappa} M_{\kappa}(x)
$$

By the discrete orthogonality property of orthogonal polynomials,

$$
a_{\kappa}=h_{\kappa}^{-1} \sum_{i=1}^{n} \psi_{i} M_{\kappa}\left(x_{i}\right) g\left(x_{i}\right), \quad \kappa=0,1, \ldots, n-1,
$$

where $\psi_{i}$ is the Christoffel number corresponding to $x_{i}$. Again, multiplying (6.1) through by $e^{i \omega x} /(x-\tau)$ and integrating over $(-1,1)$ in the CPV sense yields

$$
I(\tau, \omega)=\sum_{\kappa=0}^{n-1} a_{\kappa} Z_{\kappa}(\tau, \omega)+E_{n}^{*}(\tau, \omega)
$$

The sum in (6.2) is most effectively evaluated by Clenshaw's algorithm [16]. 
7. Bound on $E_{n}(\tau, \omega)$. Taking $M_{r}$ as the Legendre polynomials $P_{r}$, we express the quadrature error $E_{n}(\tau, \omega)$ as [cf. (2.8)]

$$
E_{n}(\tau, \omega)=\frac{1}{\pi i} \int_{C} \frac{g(z) d z}{P_{n}(z)(z-\tau)} \cdot \frac{1}{2} \int_{-1}^{1} \frac{e^{i \omega x} P_{n}(x) d x}{z-x} .
$$

Since $\left|P_{n}(x)\right| \leqslant 1,-1 \leqslant x \leqslant 1$, then

$$
\frac{1}{2}\left|\int_{-1}^{1} \frac{e^{i \omega x} P_{n}(x) d x}{z-x}\right| \leqslant \frac{1}{d(C, I)},
$$

where $d(C, I)$ is the minimum distance between $C$ and $I=[-1,1]$.

From (7.1), and in view of (7.2),

$$
E_{n}(\tau, \omega) \leqslant \frac{1}{\pi} \cdot \frac{1}{d(C, I)} \int_{C} \frac{|g(z)|}{\left|P_{n}(z)\right|} \frac{|d z|}{|z-\tau|} .
$$

Let $C_{\delta}$ be the ellipse, $z=\frac{1}{2}\left(\zeta+\zeta^{-1}\right), \zeta=\delta e^{i \theta}, 0 \leqslant \theta \leqslant 2 \pi$. For $z \in C_{\delta}$, Kambo [10] has shown that

$$
\left|P_{n}(z)\right| \geqslant \frac{\delta^{n}\left(\delta^{2}-2\right)((2 n) !)}{\left(\delta^{2}-1\right) 2^{2 n}(n !)^{2}}, \quad \delta>\sqrt{2} .
$$

Now it can be shown that

$$
\frac{1}{d\left(C_{\delta}, I\right)} \leqslant \frac{2 \delta}{(\delta-1)^{2}}
$$

and

$$
\frac{1}{d\left(C_{\delta}, \tau\right)} \leqslant \frac{2 \delta}{\delta^{2}+1-\tau} .
$$

Thus, by introducing (7.4), (7.5), and (7.6) into (7.3), we have the upper bound

$$
\left|E_{n}(\tau, \omega)\right| \leqslant \frac{1}{\pi} \cdot \frac{2^{2 n+2}(\delta+1)(n !)^{2} M(\delta) l(\delta)}{(\delta-1)\left(\delta^{2}-2\right) \delta^{n-2}\left(\delta^{2}+1-\tau\right)(2 n) !},
$$

where $M(\delta)=\max _{z \in C_{\delta}}|g(z)|$ and $l(\delta)=$ length of the ellipse $C_{\delta}$.

8. Numerical Examples. All computations in this section have been performed in single-precision arithmetic (14 significant decimal digits) on the Cyber 170-720.

We illustrate our quadrature formulae with the following problems.

$$
\operatorname{Re} I\left(\frac{1}{2}, 3 \pi\right)=\int_{-1}^{1} \frac{\log (x+2) \cos 3 \pi x d x}{x-\frac{1}{2}} .
$$

Applying the rule (4.3) to this integral we obtain the following results for various $n$.

\begin{tabular}{|c|c|}
\hline$n$ & $\operatorname{Re} I_{n}\left(\frac{1}{2}, 3 \pi\right)$ \\
\hline 5 & 2.90788246006 \\
\hline 8 & 2.907871430437 \\
\hline 10 & 2.907871888962 \\
\hline 13 & 2.907871878184 \\
\hline 16 & 2.907871878184 \\
\hline
\end{tabular}


Though the exact answer is not known, the value in the last line is thought to be correct to the number of figures shown.

(b)

$$
\begin{aligned}
\operatorname{Im} I(-.13,10) & =\int_{-1}^{1} \frac{\sinh x \sin 10 x d x}{x+.13} \\
& =\frac{1}{2} \operatorname{Im} \int_{-1}^{1}\left(\frac{e^{z x}}{x+.13}-\frac{e^{-\bar{z} x}}{x+.13}\right) d x, \\
& z=1+10 i, i=\sqrt{-1} .
\end{aligned}
$$

Similarly, on applying (4.3) to the integral we obtain:

\begin{tabular}{|r|c|}
\hline$n$ & $\operatorname{Im} I_{n}(-.13,10)$ \\
\hline 5 & -.1363282406365 \\
\hline 8 & -.1363278661647 \\
\hline 10 & -.1363278661648 \\
\hline 16 & -.1363278661646 \\
\hline 20 & -.1363278661646 \\
\hline
\end{tabular}

Exact: -.1363278661646

The exact value of the integral is obtained after a simple transformation on the last integral, and then using the results in [8, Eqs. $(2.325$, no. $1,8.214$, no. 2$)]$.

For $n=5$, the error bound (7.7) with $\delta=10$ gives $1.58 \times 10^{-3}$, the actual error being $3.74 \times 10^{-7}$.

$$
\operatorname{Im} I(0,12)=\int_{-1}^{1} \frac{e^{t} \sin 12 t d t}{t}
$$

$$
=2 \operatorname{Si}(12)-2 \sum_{\kappa=1}^{\infty} \frac{1}{(2 \kappa) !} \sum_{j=0}^{2 \kappa-1} j ! \frac{\left(\begin{array}{c}
2 \kappa-1 \\
j
\end{array}\right)}{12^{j+1}} \cos \left(12+\frac{\pi}{2} j\right) .
$$

Taking the series expansion of the exponential function and using the results in $[8$, Eqs. $(2.633$, no. $1 ; 2.641$, no. 1$)$ ] we obtain the above analytical expression for the integral.

Choosing odd values of $n$ in the evaluation of this integral by formula (4.3) leads to the case of Section 5 in which $x_{(n+1) / 2}=\tau=0$. Thus, by applying (5.10) we obtain for this integral the following results:

\begin{tabular}{|r|c|}
\hline$n$ & $\operatorname{Im} \tilde{I}_{n}(0,12)$ \\
\hline 7 & 2.929140006753 \\
\hline 9 & 2.929140053492 \\
\hline 11 & 2.929140054094 \\
\hline 13 & 2.929140054093 \\
\hline 15 & 2.929140054093 \\
\hline
\end{tabular}

Exact: 2.929140054093 
For $n=5$, the error bound (7.7) with $\delta=10$ gives $1.58 \times 10^{-3}$, the actual error being $3.74 \times 10^{-7}$.

Acknowledgment. I wish to express my gratitude to Professor Walter Gautschi and the referee for their helpful comments and suggestions which have greatly improved the original manuscript.

Department of Mathematics

University of Calabar

Calabar, Nigeria

1. M. Abramowitz \& I. A. Stegun, Handbook of Mathematical Functions, with Formulas, Graphs, and Mathematical Tables, Dover, New York, 1970.

2. N. S. Bahvalov \& L. G. VASIL'EVA, "Evaluation of the integrals of oscillatory functions by interpolation at nodes of Gaussian quadratures," Ž. Vyčisl. Mat. i Mat. Fiz., v. 8, 1968, pp. 175-181. (Russian)

3. M. M. Chawla \& N. Jayarajan, "Quadrature formulas for Cauchy principal value integrals," Computing, v. 15,1975 , pp. 347-355.

4. P. J. Davis \& P. Rabinowitz, Methods of Numerical Integration, Academic Press, New York, 1975.

5. D. Elliott \& D. F. PAGET, "Gauss-type quadrature rules for Cauchy principal value integrals," Math. Comp., v. 33, 1979, pp. 301-309.

6. L. N. G. FILON, “On a quadrature formula for trigonometric integrals,” Proc. Roy. Soc. Edinburgh, v. 49,1929, pp. $38-47$.

7. W. Gautschi, “A survey of Gauss-Christoffel quadrature formulae," in E. B. Christoffel (P. L. Butzer and F. Fehér, eds.), Birkhäuser Verlag, Basel, 1981, pp. 73-147.

8. I. S. Gradshteyn \& I. M. RyzhiK, Table of Integrals, Series, and Products, Academic Press, New York, 1980.

9. D. B. Hunter, "Some Gauss-type formulae for the evaluation of Cauchy principal values of integrals," Numer. Math., v. 19, 1972, pp. 419-424.

10. N. S. Kambo, "Error of the Newton-Cotes and Gauss-Legendre quadrature formulas," Math. Comp., v. 24, 1970, pp. 261-269.

11. S. Kumar, “A note on quadrature formulae for Cauchy principal value integrals," J. Inst. Math. Appl., v. 26, 1980, pp. 447-451.

12. R. K. Littlewood \& V. ZaKian, "Numerical evaluation of Fourier integrals," J. Inst. Math. Appl., v. 18, 1976, pp. 331-339.

13. I. M. LONGMAN, "On the numerical evaluation of Cauchy principal values of integrals," $M T A C$, v. 12, 1958, pp. 205-207.

14. Y. L. LUKE, “On the computation of oscillatory integrals, Part 2," Proc. Cambridge Philos. Soc., v. 50, 1954, pp. 264-277.

15. J. C. P. Miller, British Association for the Advancement of Science, Mathematical Tables, Volume X, Bessel Functions, Part II, Functions of Positive Integer Order, Cambridge Univ. Press, New York, 1952.

16. D. F. Paget \& D. Elliotr, "An algorithm for the numerical evaluation of certain Cauchy principal value integrals," Numer. Math., v. 19, 1972, pp. 373-385.

17. T. N. L. Patterson, "On high precision methods for the evaluation of Fourier integrals with finite and infinite limits," Numer. Math., v. 27, 1976, pp. 41-52.

18. R. Piessens \& F. Poleunis, "A numerical method for the integration of oscillatory functions," $B I T$, v. 11, 1971, pp. 317-327.

19. R. Piessens \& M. Branders, "The evaluation and application of some modified moments," $B I T, \mathrm{v}$. 13, 1973, pp. 443-450.

20. A. Ralston \& P. Rabinowitz, A First Course in Numerical Analysis, 2nd ed., McGraw-Hill, New York, 1978.

21. G. Szegö, Orthogonal Polynomials, Amer. Math. Soc. Colloq. Publ., vol. 23, Amer. Math. Soc., Providence, R. I., 1975.

22. B. TING \& Y. L. LuKE, "Computation of integrals with oscillatory and singular integrands," Math. Comp., v. 37, 1981, pp. 169-183. 